\title{
Some Remarks on Biharmonic Elliptic Problems with a Singular Nonlinearity
}

\author{
Baishun Lai ${ }^{1,2}$ \\ ${ }^{1}$ Institute of Contemporary Mathematics, Henan University, Kaifeng 475004, China \\ ${ }^{2}$ School of Mathematics and Information Science, Henan University, Kaifeng 475004, China \\ Correspondence should be addressed to Baishun Lai; laibaishun@henu.edu.cn
}

Received 3 December 2013; Accepted 26 February 2014; Published 31 March 2014

Academic Editor: Alberto Fiorenza

Copyright (C) 2014 Baishun Lai. This is an open access article distributed under the Creative Commons Attribution License, which permits unrestricted use, distribution, and reproduction in any medium, provided the original work is properly cited.

We study the following semilinear biharmonic equation $\Delta^{2} u=\lambda / 1-u$, in $\mathbb{B}$, and $u=\partial u / \partial n=0$, on $\partial \mathbb{B}$, where $\mathbb{B}$ is the unit ball in $\mathbb{R}^{n}$ and $n$ is the exterior unit normal vector. We prove the existence of $\lambda^{*}>0$ such that for $\lambda \in\left(0, \lambda^{*}\right)$ there exists a minimal (classical) solution $\underline{u}_{\lambda}$, which satisfies $0<\underline{u}_{\lambda}<1$. In the extremal case $\lambda=\lambda^{*}$, we prove the existence of a weak solution which is the unique solution even in a very weak sense. Besides, several new difficulties arise and many problems still remain to be solved. We list those of particular interest in the final section.

\section{Introduction and Results}

In the last forty years, a great deal has been written about existence and multiplicity of solutions to nonlinear second order elliptic problems in bounded and unbounded domains of $\mathbb{R}^{n}(n \geq 2)$. Important achievements on this topic have been made by applying various combinations of analytical techniques, which include the variational and topological methods. For the latter, the fundamental tool which has been widely used is the maximum principle. However, for higher order problems, a possible failure of the maximum principle causes several technical difficulties, which attracted the interest of many researchers. In particular, recently, fourth order equations with a singular nonlinearity have been studied extensively. The motivation for considering these equations stems from a model for the steady states of a simple microelectromechanical system (MEMS) which has the general form (see, e.g., [1])

$$
\begin{aligned}
\alpha \Delta^{2} u= & \left(\beta \int_{\Omega}|\nabla u|^{2} d x+\gamma\right) \Delta u \\
& +\frac{\lambda f(x)}{(1-u)^{2}\left(1+\chi \int_{\Omega}\left(d x /(1-u)^{2}\right)\right)} \text { in } \Omega,
\end{aligned}
$$

$$
\begin{gathered}
0<u<1 \quad \text { in } \Omega, \\
u=\alpha \frac{\partial u}{\partial n}=0 \quad \text { on } \partial \Omega,
\end{gathered}
$$

where $\Delta^{2}(\cdot):=-\Delta(-\Delta)$ denotes the biharmonic operator, $\Omega \subset \mathbb{R}^{n}$ is a smooth bounded domain, $n$ denotes the outward pointing unit normal to $\partial \Omega$ and $\alpha, \beta, \gamma, \chi \geq 0$ are physically relevant constants, $f \geq 0$ represents the permittivity profile, and $\lambda>0$ is a constant which is increasing with respect to the applied voltage.

Let $\gamma=\beta=\chi=0$ and $\alpha=1, f(x) \equiv 1$ in the above model, we obtain, replacing $(1-u)^{-2}$ with $(1-u)^{-p}$.

$$
\begin{gathered}
\Delta^{2} u=\frac{\lambda}{(1-u)^{p}} \quad \text { in } \Omega, \\
0<u<1 \quad \text { in } \Omega, \\
u=\frac{\partial u}{\partial n}=0 \quad \text { on } \partial \Omega .
\end{gathered}
$$

Because of the lack of a "maximum principle," which plays such a crucial role in developing the theory for the Laplacian, 
for $\Delta^{2}$ with Dirichlet boundary condition in general domains (i.e., $\Omega \neq \mathbb{B}$ ), very little is known about $\left(P_{\lambda}\right)$. As far as we are aware, only the paper [2] studies this problem for general domains. However, if $p>1$ and $\Omega$ is a ball, $\left(P_{\lambda}\right)$ has recently been studied extensively; see, for example, [3-9] and their references. One of the reasons to study $\left(P_{\lambda}\right)$ in a ball is that a maximum principle holds in this situation; see [10], and so some tools that are well suited for the corresponding second equation can work for $\left(P_{\lambda}\right)$. The second reason is that one can easily find an explicit singular radial solution, denoted by $1-|x|^{4 /(p+1)}(p>1)$, of $\left(P_{\lambda}\right)$ for $\Omega=\mathbb{B}$ and a suitable parameter $\lambda$ which satisfy the first boundary condition but not the second. The singular radial solution, called "ghost" singular solution, plays a fundamental role to characterize the "true" singular solution; see, in particular, [4].

In this paper, we will focus essentially our attention on the case where $p=1$ and the $\Omega$ is a ball; namely,

$$
\begin{gathered}
\Delta^{2} u=\frac{\lambda}{1-u} \quad \text { in } \mathbb{B}, \\
0<u \leq 1 \quad \text { in } \mathbb{B}, \\
u=\frac{\partial u}{\partial n}=0 \quad \text { on } \partial \mathbb{B} .
\end{gathered}
$$

The corresponding second order problem, which is related to the general study of singularities of minimal hypersurfaces of Euclidean space, has been studied by Meadows; see [11]. In that case, however, the start point was an explicit singular solution (i.e., $u_{s}(r)=1-r^{2}$ ) with parameter $\lambda=n-1$. When turning to the biharmonic problem $(1)_{\lambda}$, one cannot find any explicit singular solution even "ghost" singular solution, which causes several technical difficulties. The first purpose of the present paper is to extend $(1)_{\lambda}$ some well-known results relative to $\left(P_{\lambda}\right)$. The second (and perhaps the most important) purpose of the present paper is to emphasize some striking differences between $(1)_{\lambda}$ and $\left(P_{\lambda}\right)$.

1.1. Preliminaries. Besides classical solution that is $u \in$ $C^{4}(\overline{\mathbb{B}})$ which satisfies $(1)_{\lambda}$, let us introduce the class of weak solutions we will be dealing with. We denote by $H_{0}^{2}(\mathbb{B})$ the usual Sobolev space which can be defined by completion as follows:

$$
H_{0}^{2}(\mathbb{B}):=\operatorname{cl}\left\{u \in C_{0}^{\infty}(\mathbb{B}):\|\Delta u\|_{2}<\infty\right\}
$$

which is a Hilbert space endowed with the scalar product:

$$
(u, v)_{\mathrm{H}_{0}^{2}(\mathbb{B})}:=\int_{\mathbb{B}} \Delta u \Delta v d x
$$

Definition 1. One says that $u \in L^{1}(\mathbb{B})$ is a weak solution of $(1)_{\lambda}$ provided $0 \leq u \leq 1$ almost everywhere, $1 /(1-u) \epsilon$ $L^{1}(\mathbb{B})$, and

$$
\int_{\mathbb{B}} u \Delta^{2} \varphi d x=\lambda \int_{\mathbb{B}} \frac{\varphi}{(1-u)} d x, \quad \forall \varphi \in C^{4}(\overline{\mathbb{B}}) \cap H_{0}^{2}(\mathbb{B}) .
$$

When in (3) the equality is replaced by the inequality $\geq$ (resp. $\leq$ ) and $\varphi \geq 0$, we say that $u$ is a weak supersolution (resp., weak subsolution) of (3) provided the following boundary conditions are satisfied: $u=0$ (resp. $=)$ and $\partial u / \partial n \leq 0$ (resp. $\geq$ ) on $\partial \mathbb{B}$.

Definition 2. One calls a solution $u$ of $(1)_{\lambda}$ minimal if $u \leq v$ almost everywhere in $\mathbb{B}$ for any further solution $v$ of $(1)_{\lambda}$.

If $u$ is a classical solution of $(1)_{\lambda}$, then the linearized operator at $u$ turns out to be well defined:

$$
L_{u}:=\Delta^{2}-\frac{\lambda}{(1-u)^{2}} .
$$

which yields the following notion of stability.

Definition 3. A classical solution $u$ of $(1)_{\lambda}$ is semistable provided

$$
\begin{aligned}
\mu_{1}(u) & =\inf \left\{\int_{\mathbb{B}}(\Delta \varphi)^{2}-\frac{\lambda \varphi^{2}}{(1-u)^{2}}: \phi \in H_{0}^{2}(\mathbb{B}),\|\phi\|_{L^{2}}=1\right\} . \\
& \geq 0
\end{aligned}
$$

If $\mu_{1}(u)>0$, we say that $u$ is stable.

As far as we are concerned with weak solutions, the linearized operator is no longer well defined; however, we introduce the following weaker notion of stability.

Definition 4. A weak solution $u$ to $(1)_{\lambda}$ is said to be weakly stable if $1 /(1-u)^{2} \in L^{1}(\mathbb{B})$ and the following holds:

$$
\int_{\mathbb{B}}|\Delta \varphi|^{2} d x \geq \int_{\mathbb{B}} \frac{\lambda \varphi^{2}}{(1-u)^{2}} d x, \quad \varphi \in H_{0}^{2}(\mathbb{B}), \varphi \geq 0 .
$$

According to the class of solutions which we consider, let us introduce the following values:

$$
\begin{aligned}
\lambda^{*} & :=\sup \left\{\lambda \geq 0:(1)_{\lambda} \text { possesses a weak solution }\right\} \\
\lambda_{*} & :=\sup \left\{\lambda \geq 0:(1)_{\lambda} \text { possesses a classical solution }\right\} .
\end{aligned}
$$

Remark 5. Clearly, a classical solution is also a weak solution, so that one has $\lambda_{*} \leq \lambda^{*}$. Moreover, by standard elliptic regularity theory for the biharmonic operator [12], any weak solution of $(1)_{\lambda}$ which satisfies $\left\|u_{\lambda}\right\|_{L^{\infty}}<1$ turns out to be smooth.

Besides, we give a notion of $H_{0}^{2}(\mathbb{B})$-weak solutions, which is an intermediate class between classical and weak solutions.

Definition 6. One says that $u$ is a $H_{0}^{2}(\mathbb{B})$-weak solution of (3) if $(1-u)^{-1} \in L^{1}(\mathbb{B})$ and if

$$
\int_{\mathbb{B}} \Delta u \Delta \phi=\lambda \int_{\mathbb{B}} \phi(1-u)^{-1}, \quad \forall \phi \in C^{4}(\overline{\mathbb{B}}) \cap H_{0}^{2}(\mathbb{B}) .
$$


One says that $u$ is a $H_{0}^{2}(\mathbb{B})$-weak supersolution $\left(\right.$ resp. $H_{0}^{2}(\mathbb{B})$ weak subsolution) of $(1)_{\lambda}$ if for $\phi \geq 0$ the equality is replaced with $\geq($ resp. $\leq)$ and $u \geq 0$ (resp. $\leq), \partial u / \partial n \leq 0$ (resp. $\geq$ ) on $\partial \mathbb{B}$.

1.2. Main Results. In order to state our results, we denote by $v_{1}$ the first eigenvalue of the biharmonic operator on $\mathbb{B}$ with Dirichlet boundary conditions, which is characterized variationally as follows:

$$
\nu_{1}:=\inf \left\{\int_{\mathbb{B}}|\Delta u|^{2} d x: u \in H_{0}^{2}(\mathbb{B}),\|u\|_{L^{2}}=1\right\} .
$$

It is well known that $v_{1}>0$ is simple, isolated and that the corresponding eigenfunctions $\psi>0$, spherically symmetric, radially decreasing and do not change sign.

We may now state the following theorem.

Theorem 7. There exists $\lambda_{*}>0$ such that, for $0<\lambda<\lambda_{*},(1)_{\lambda}$ possesses a minimal classical solution, denoted by $\underline{u}_{\lambda}$, which is positive and stable. Moreover, $\lambda_{*}$ satisfies the following bounds:

$$
\max \{4 n(n-2), 2 n(n+2)\} \leq \lambda_{*} \leq \frac{\nu_{1}}{4} \text {. }
$$

It is remarkable that at $\lambda_{*}$ there is an immediate switch from existence of regular minimal solutions to nonexistence of any (even singular) solution. The only possibly singular minimal solution corresponds to $\lambda=\lambda_{*}$. This result is known from [13] for the corresponding second order problems, but the method used there may not be carried over to fourth order problems. Nevertheless, the result extends to biharmonic case in the following theorem.

Theorem 8. The following holds:

$$
\lambda_{*}=\lambda^{*} \text {. }
$$

In particular, for $\lambda>\lambda^{*}$ there are no solutions, even in the weak sense. Furthermore, for almost every $x \in \mathbb{B}$, there exists

$$
u^{*}(x):=\lim _{\lambda \rightarrow \lambda^{*}} \underline{u}_{\lambda}(x)
$$

and $u^{*}(x)$ is a weakly stable $H_{0}^{2}(\mathbb{B})$-weak solution of $(1)_{\lambda^{*}}$, which is called the extremal solution.

If $n \leq 4$, then the extremal solution $u^{*}$ of $(1)_{\lambda}$ is smooth; that is, $u^{*}=\lim _{\lambda \rightarrow \lambda^{*}} \underline{u}_{\lambda}(x)$ exists in the topology of $C^{4}(\mathbb{B})$. It is the unique regular solution to $(1)_{\lambda^{*}}$.

From the above theorem, we note that the function $u^{*}$ exists in any dimension and does solve $(1)_{\lambda^{*}}$ in the $H_{0}^{2}(\mathbb{B})$ weak sense and it is a classical solution in dimensions $1 \leq n \leq$ 4. This will allow us to start another branch of nonminimal (unstable) solutions. Besides, inspired by $[3,14,15]$, we get the following uniqueness of the extremal solution of $(1)_{\lambda^{*}}$, which gives Theorem 9 .

Theorem 9. Let $v$ be a weak supersolution of $(1)_{\lambda}$ with parameter $\lambda^{*}$. Then, $v=u^{*}$; in particular, $(1)_{\lambda^{*}}$ has a unique weak solution.
From this theorem, we know that there are no strict supersolutions to $(1)_{\lambda^{*}}$.

Corollary 10. Let $u_{\lambda} \in H_{0}^{2}(\mathbb{B})$ be a weak solution of $(1)_{\lambda}$ such that $\left\|u_{\lambda}\right\|_{L^{\infty}}=1$. Then, $u_{\lambda}$ is weakly stable if and only if $\lambda=\lambda^{*}$ and $u_{\lambda}=u^{*}$.

We may also characterize the uniform convergence to 0 of $\underline{u}_{\lambda}$ as $\lambda \rightarrow 0$ by giving the precise rate of its extinction.

Theorem 11. For all $\lambda \in\left(0, \lambda^{*}\right)$, let $\underline{u}_{\lambda}$ be the minimal solution of $(1)_{\lambda}$ and let

$$
V_{\lambda}(x)=\frac{\lambda}{8 n(n+2)}\left[1-|x|^{2}\right]^{2} .
$$

Then, $\underline{u}_{\lambda}>V_{\lambda}(x)$ for all $\lambda<\lambda^{*}$ and all $|x|<1$, and

$$
\lim _{\lambda \rightarrow 0} \frac{\underline{u}_{\lambda}}{V_{\lambda}(x)}=1 \quad \text { uniformly with respect to } x \in \mathbb{B} \text {. }
$$

1.3. Key Ingredients. Now, we give some comparison principles which will be used throughout the paper.

Lemma 12 (Boggio's principle, [10]). If $u \in C^{4}\left(\overline{\mathbb{B}}_{R}\right)$ satisfies

$$
\begin{gathered}
\Delta^{2} u \geq 0 \quad \text { in } \quad \mathbb{B}_{R}, \\
u=\frac{\partial u}{\partial n}=0 \quad \text { on } \partial \mathbb{B}_{R},
\end{gathered}
$$

then $u \geq 0$ in $\mathbb{B}_{R}$.

Lemma 13. Let $u \in L^{1}\left(\mathbb{B}_{R}\right)$ and suppose that

$$
\int_{\mathbb{B}_{R}} u \Delta^{2} \varphi \geq 0
$$

for all $\varphi \in C^{4}\left(\overline{\mathbb{B}}_{R}\right)$ such that $\varphi \geq 0$ in $\mathbb{B}_{R},\left.\varphi\right|_{\partial \mathbb{B}_{R}}=$ $\left.(\partial \varphi / \partial n)\right|_{\partial \mathbb{B}_{R}}=0$. Then, $u \geq 0$ in $\mathbb{B}_{R}$. Moreover, $u \equiv 0$ or $u>0$ almost everywhere, in $\mathbb{B}_{R}$.

For a proof, see Lemma 17 in [16]. From this lemma, we know that any solution of $(1)_{\lambda}$ is necessarily positive almost everywhere inside the ball.

Lemma 14. If $u \in H^{2}\left(\mathbb{B}_{R}\right)$ is radial, $\Delta^{2} u \geq 0$ in $\mathbb{B}_{R}$ in the weak sense, that is,

$$
\int_{\mathbb{B}_{R}} \Delta u \Delta \varphi \geq 0 \quad \forall \varphi \in C_{0}^{\infty}\left(\mathbb{B}_{R}\right), \varphi \geq 0,
$$

and $\left.u\right|_{\partial \mathbb{B}_{R}} \geq 0,\left.(\partial u / \partial n)\right|_{\partial \mathbb{B}_{R}} \leq 0$, then $u \geq 0$ in $\mathbb{B}_{R}$.

Proof. The proof is standard; see [15]; we give a proof here for the sake of completeness. We only deal with the case $R=1$ for simplicity. Solve

$$
\begin{aligned}
\Delta^{2} u_{1} & =\Delta^{2} u \quad \text { in } \mathbb{B}, \\
u_{1} & =\frac{\partial u_{1}}{\partial n}=0 \quad \text { on } \partial \mathbb{B}
\end{aligned}
$$


in the sense that $u_{1} \in H_{0}^{2}(\mathbb{B})$ and $\int_{\mathbb{B}} \Delta u_{1} \Delta \varphi=\int_{\mathbb{B}} \Delta u \Delta \varphi$ for all $\varphi \in C_{0}^{\infty}(\mathbb{B})$. Then, $u_{1} \geq 0$ in $\mathbb{B}$ by Lemma 24 .

Let $u_{2}=u-u_{1}$ so that $\Delta^{2} u_{2}=0$ in $\mathbb{B}$. Define $f=\Delta u_{2}$. Then, $\Delta f=0$ in $\mathbb{B}$ and since $f$ is radial we find that $f$ is a constant. It follows that $u_{2}=a r^{2}+b$. Using the boundary conditions, we deduce $a+b \geq 0$ and $a \leq 0$, which imply $u_{2} \geq 0$.

Lemma 15. Let $f \in L^{1}\left(\mathbb{B}_{R}\right), f \geq 0$ almost everywhere. Then, there exists a unique $u \in L^{1}\left(\mathbb{B}_{R}\right)$ such that $u \geq 0$ and

$$
\int_{\mathbb{B}_{R}} u \Delta^{2} \varphi=\int_{\mathbb{B}_{R}} f \varphi, \quad \varphi \in C^{4}\left(\overline{\mathbb{B}}_{R}\right) \cap H_{0}^{2}\left(\mathbb{B}_{R}\right) .
$$

Moreover, there exists $C>0$ which does not depend on $f$ such that $\|u\|_{1} \leq C\|f\|_{1}$.

Proof. The proof is standard; see [16]; we give a proof here for the sake of completeness. The uniqueness is clear. Indeed, let $v_{1}$ and $v_{2}$ be two solutions of (19). Then, $v=v_{1}-v_{2}$ satisfies

$$
\int_{\mathbb{B}} v \Delta^{2} \varphi=0 \quad \varphi \in C^{4}\left(\overline{\mathbb{B}}_{R}\right) \cap H_{0}^{2}\left(\mathbb{B}_{R}\right) .
$$

Given any $\zeta \in C_{0}^{\infty}(\mathbb{B})$, let $\varphi$ be the solution of

$$
\begin{aligned}
\Delta^{2} \varphi & =\zeta \quad \text { in } \mathbb{B}, \\
\varphi & =\frac{\partial \varphi}{\partial n}=0 \text { on } \partial \mathbb{B} .
\end{aligned}
$$

It follows that

$$
\int_{\mathbb{B}} v \zeta=0
$$

Since $\zeta$ is arbitrary, we deduce that $v=0$.

For the existence, given an integer $k \geq 0$, we set $f_{k}=$ $\min \{f(x), k\}$, so that $f_{k} \rightarrow f$ as $k \rightarrow \infty$ in $L^{1}(\mathbb{B})$. Let $v_{k}$ be the solution of

$$
\begin{aligned}
\Delta^{2} v_{k} & =f_{k} \quad \text { in } \mathbb{B}, \\
v_{k} & =\frac{\partial v_{k}}{\partial n}=0 \quad \text { on } \partial \mathbb{B} .
\end{aligned}
$$

The sequence $\left(v_{k}\right)_{k \geq 0}$ is clearly monotone nondecreasing. It is also a Cauchy sequence in $L^{1}(\mathbb{B})$ since

$$
\int_{\mathbb{B}}\left(v_{k}-v_{l}\right)=\int_{\mathbb{B}}\left(f_{k}-f_{l}\right) \zeta_{0},
$$

where $\zeta_{0}$ is defined by

$$
\begin{array}{cc}
\Delta^{2} \zeta_{0}=1 & \text { in } \mathbb{B}, \\
\zeta_{0}=\frac{\partial \zeta_{0}}{\partial n}=0 & \text { on } \partial \mathbb{B} .
\end{array}
$$

Hence,

$$
\int_{\mathbb{B}}\left|v_{k}-v_{l}\right| \leq C \int_{\mathbb{B}}\left|f_{k}-f_{l}\right| d x .
$$

Passing to the limit in (23) (after multiplication by $\varphi$ ), we obtain (19) and $u \geq 0$ according to Lemma 13 . Finally, taking $\varphi=\zeta_{0}$ in (19), we obtain

$$
\|v\|_{L^{1}}=\int_{\mathbb{B}} v=\int_{\mathbb{B}} f \zeta_{0} \leq C\|f\|_{L^{1}},
$$

and the proof is completed.

Proposition 16. Assume the existence of a weak supersolution $U$ of $(1)_{\lambda}$. Then, there exists a weak solution $u$ of $(1)_{\lambda}$ so that $0 \leq u \leq U$ almost everywhere in $\mathbb{B}$.

Proof. By means of a standard monotone iteration argument, set $u_{0}:=U$ and define recursively $u_{n+1} \in L^{1}(\mathbb{B})$ as the unique solution of

$$
\int_{\mathbb{B}} u_{n+1} \Delta^{2} \varphi d x=\lambda \int_{\mathbb{B}} \frac{\varphi}{\left(1-u_{n}\right)^{2}} d x, \quad \varphi \in C^{4}(\overline{\mathbb{B}}) \cap H_{0}^{2}(\mathbb{B}) ;
$$

then we have

$$
\int_{\mathbb{B}}\left(u_{n}-u_{n+1}\right) \Delta^{2} \varphi d x \geq 0, \quad \varphi \in C^{4}(\overline{\mathbb{B}}) \cap H_{0}^{2}(\mathbb{B}),
$$

and Lemma 24 yields $0 \leq u_{n+1} \leq u_{n}<U(x)$ almost everywhere for all $n \in \mathbb{N}$. Since

$$
\left(1-u_{n}\right)^{-1} \leq(1-U)^{-1} \in L^{1}(\mathbb{B}),
$$

the claim follows from the Lebesgue convergence theorem.

We complete these preliminary results by proving a key lemma which provides a comparison principle.

Lemma 17. Assume $u_{1}$ is a weakly stable $H_{0}^{2}(\mathbb{B})$-weak subsolution of $(1)_{\lambda}$ and $u_{2}$ is $H_{0}^{2}(\mathbb{B})$-weak supersolution of $(1)_{\lambda}$. Then,

(1) $u_{1} \leq u_{2}$ almost everywhere in $\mathbb{B}$;

(2) if $u$ is a classical solution such that $\mu_{1}(u)=0$ and $U$ is any classical supersolution of $(1)_{\lambda}$, then $u \equiv U$.

Proof. (1) Define $\omega:=u_{1}-u_{2}$. Then, by the Moreau decomposition [9] for the biharmonic operator, there exists $\omega_{1}, \omega_{2} \in H_{0}^{2}(\mathbb{B})$, with $\omega=\omega_{1}+\omega_{2}, \omega_{1} \geq 0$ almost everywhere, $\Delta^{2} \omega_{2} \leq 0$ in the $H_{0}^{2}(\mathbb{B})$-weak sense, and

$$
\int_{\mathbb{B}} \Delta \omega_{1} \Delta \omega_{2}=0
$$

By Lemma 12, we have that $\omega_{2} \leq 0$ almost everywhere in $\mathbb{B}$. Given now $0 \leq \varphi \in C_{0}^{\infty}(\mathbb{B})$, we have that

$$
\int_{\mathbb{B}} \Delta \omega \Delta \varphi \leq \lambda \int_{\mathbb{B}}\left(f\left(u_{1}\right)-f\left(u_{2}\right)\right) \varphi,
$$

where $f(u)=(1-u)^{-1}$. Since $u_{1}$ is stable, one has

$$
\begin{aligned}
\lambda \int_{\mathbb{B}} f^{\prime}\left(u_{1}\right) \omega_{1}^{2} & \leq \lambda \int_{\mathbb{B}}\left(\Delta \omega_{1}\right)^{2} \\
& =\lambda \int_{\mathbb{B}} \Delta \omega \Delta \omega_{1} \leq \lambda \int_{\mathbb{B}}\left(f\left(u_{1}\right)-f\left(u_{2}\right)\right) \omega_{1} .
\end{aligned}
$$


Since $\omega_{1} \geq \omega$, one also has

$$
\int_{\mathbb{B}} f^{\prime}\left(u_{1}\right) \omega \omega_{1} \leq \int_{\mathbb{B}}\left(f\left(u_{1}\right)-f\left(u_{2}\right)\right) \omega_{1},
$$

which once rearranged gives

$$
\int_{\mathbb{B}} \tilde{f} \omega_{1} \geq 0
$$

where $\tilde{f}\left(u_{1}\right)=f\left(u_{1}\right)-f\left(u_{2}\right)-f^{\prime}\left(u_{1}\right)\left(u_{1}-u_{2}\right)$. The strict convexity of $f$ gives $\widetilde{f} \leq 0$ and $\widetilde{f}<0$ whenever $u_{1} \neq u_{2}$. Since $\omega_{1} \geq 0$ almost everywhere in $\mathbb{B}$, one sees that $\omega \leq 0$ almost everywhere in $\mathbb{B}$. The inequality $u_{1} \leq u_{2}$ almost everywhere in $\mathbb{B}$ is then established.

(2) Let $\varphi>0$ be the first eigenfunction of $\Delta^{2}-\lambda f^{\prime}(u)$ in $H_{0}^{2}(\mathbb{B})$; we, now, for $0 \leq t \leq 1$, define

$$
g(t)=\int_{\mathbb{B}} \Delta(t U+(1-t) u) \Delta \phi-\lambda \int_{\mathbb{B}} f(t U+(1-t) u) \phi,
$$

where $\phi$ is the above first eigenfunction. Since $f$ is convex, one sees that

$$
\begin{aligned}
g(t) & \geq \lambda \int_{\mathbb{B}}[t f(U)+(1-t) f(u)-f(t U+(1-t) u)] \phi \\
& \geq 0
\end{aligned}
$$

for every $t \geq 0$. Since $g(0)=0$ and

$$
g^{\prime}(0)=\int_{\mathbb{B}} \Delta(U-u) \Delta \phi-\lambda f^{\prime}(u)(U-u) \phi=0,
$$

we get that

$$
g^{\prime \prime}(0)=-\lambda \int_{\mathbb{B}} f^{\prime \prime}(u)(U-u)^{2} \phi \geq 0
$$

Since $f^{\prime \prime}(u) \phi>0$ in $\mathbb{B}$, we finally get that $U=u$ almost everywhere in $\mathbb{B}$.

\section{Existence Results: Proofs of Theorems 7 and 8}

2.1. The Branch of Minimal Solutions. Let us define

$$
\Lambda:=\left\{\lambda \geq 0:(1)_{\lambda}\right.
$$

has a classical solution with parameter $\lambda\}$.

Proposition 18. For all $0 \leq \lambda<\lambda_{*}$, there exists a minimal classical solution $\underline{u}_{\lambda}$ of $(1)_{\lambda}$ which is smooth and stable. Moreover,

(i) the map $\lambda \rightarrow \underline{u}_{\lambda}$, for $\lambda \in\left(0, \lambda_{*}\right)$, is differentiable and strictly increasing;

(ii) the map $\lambda \rightarrow \mu_{1}\left(\underline{u}_{\lambda}\right)$ is decreasing on $\left(0, \lambda_{*}\right)$;

(iii) let $\tilde{u}_{\lambda}$ be a regular solution of $(1)_{\lambda}$ for $\lambda \in\left(0, \lambda_{*}\right)$;; if $\tilde{u}_{\lambda}$ is not the minimal solution, then $\mu_{1}\left(\tilde{u}_{\lambda}\right)<0$.
Proof. First, we show that $\Lambda$ does not consist of just $\lambda=0$. To this end, let $\psi_{R}$ be the first eigenfunction of the biharmonic operator subject to Dirichlet boundary conditions on $\mathbb{B}_{R} \supset \mathbb{B}$ which we normalize by $\sup _{\mathbb{B}_{R}} \Psi_{R}=1$ and let $\nu_{R}>0$ be the corresponding eigenvalue. Next, we are going to prove that for $\theta \in(0,1)$ the function $\psi=\theta \psi_{R}$ is a supersolution of $(1)_{\lambda}$ as long as $\lambda$ is sufficiently small. We have

$$
0<1-\theta \psi_{R}<1, \quad \text { in } \mathbb{B}
$$

moreover,

$$
\Delta^{2} \psi=\nu_{R} \theta \psi_{R} \geq \frac{\lambda}{1-\theta \psi_{R}}=\frac{\lambda}{1-\psi}
$$

provided that

$$
v_{R} \theta \psi_{R}\left(1-\theta \psi_{R}\right) \geq \lambda
$$

Notice that

$$
0<s_{1}:=\inf _{x \in \mathbb{B}} \psi<s_{2}:=\sup _{x \in \mathbb{B}} \psi<1
$$

and that $\partial \psi / \partial n<0$ on $\partial \mathbb{B}$. Thus, looking at the function $g(s)=s(1-s)$, for $s \in\left[s_{1}, s_{2}\right]$, it is easily seen that we can choose $\lambda>0$ sufficiently small such that

$$
v_{R} \inf _{x \in \mathbb{B}} g(\theta \psi(x))>\lambda .
$$

Since $\underline{u} \equiv 0$ is a subsolution of $(1)_{\lambda}$, the classical subsuper solution theorem provides a classical solution $u_{\lambda}$ to $(1)_{\lambda}$. With such function $u_{\lambda}$, we can use the Boggio principle to show straightforwardly that the iterative scheme

$$
\begin{aligned}
\Delta^{2} u_{n, \lambda} & =\frac{\lambda}{\left(1-u_{n-1, \lambda}\right)} \quad \text { in } \mathbb{B}, \\
u_{n, \lambda} & =\frac{\partial u_{n, \lambda}}{\partial n}=0 \quad \text { in } \partial \mathbb{B}, \\
u_{0, \lambda} & =0 \quad \text { in } \mathbb{B}
\end{aligned}
$$

gives rise to a monotone sequence $\left\{u_{n, \lambda}\right\}$ satisfying

$$
0=u_{0, \lambda} \leq u_{1, \lambda} \leq \cdots \leq u_{n-1, \lambda} \leq \cdots \leq u_{\lambda}<1
$$

for all $n \in \mathbb{N}$. Therefore, the minimal solution $\underline{u}_{\lambda}$ is obtained as the increasing limit

$$
\underline{u}_{\lambda}(x):=\lim _{n \rightarrow \infty} u_{n, \lambda} .
$$

Again from the Boggio positivity preserving property (Lemma 12), we obtain $0<\underline{u}_{\lambda}<1$; in particular, from standard elliptic regularity theory for the biharmonic operator, it follows that $\underline{u}_{\lambda}(x)$ is smooth. In order to prove stability, let us argue as follows: set

$$
\lambda_{* *}:=\sup \left\{\lambda \in\left(0, \lambda_{*}\right): \mu_{1}\left(\underline{u}_{\lambda}\right)>0\right\} ;
$$

clearly $\lambda_{* *} \leq \lambda_{*}$. Now, suppose by contradiction that $\lambda_{* *}<$ $\lambda_{*}$ and let $\varepsilon>0$ sufficiently small such that $\lambda_{* *}+\varepsilon<\lambda_{*}$ 
and $v_{\lambda_{* *}+\varepsilon}$ is the corresponding minimal solution. By the definition and left continuity of the map $\lambda \rightarrow \mu_{1}\left(\underline{u}_{\lambda}\right)$, we have necessarily $\mu_{1}\left(\underline{u}_{\lambda_{* *}}\right)=0$. Since $v_{\lambda_{* *}+\varepsilon}$ is a supersolution of $(1)_{\lambda_{* *}}$, by Lemma 17 , we get $v_{\lambda_{* *}+\varepsilon}=\underline{u}_{\lambda_{* *}}$ and thus $\varepsilon=0$, a contradiction.

Since each $\underline{u}_{\lambda}$ is stable, then by setting $F\left(\underline{u}_{\lambda}, \lambda\right):=\Delta^{2}-$ $\lambda /\left(1-\underline{u}_{\lambda}\right)$, we get that $F_{\underline{u}_{\lambda}}\left(\underline{u}_{\lambda}, \lambda\right)$ is invertible for $0<\lambda<\lambda_{*}$. It then follows from Implicit Function Theorem that $\underline{u}_{\lambda}(x)$ is differentiable with respect to $\lambda$.

Now, we prove that the map $\lambda \rightarrow \underline{u} \lambda$ is strictly increasing on $\left(0, \lambda_{*}\right)$. Consider $\lambda_{1}<\lambda_{2}<\lambda_{*}$ and their corresponding minimal positive solutions $\underline{u}_{\lambda_{1}}$ and $\underline{u}_{\lambda_{2}}$, and let $u^{*}$ be a solution for $(1)_{\lambda_{2}}$. The same as the above iterative scheme, we have

$$
\underline{u}_{\lambda_{1}}=\lim _{n \rightarrow \infty} u_{n}\left(\lambda_{1} ; x\right) \leq u^{*} \quad \text { in } \mathbb{B},
$$

and in particular $\underline{u}_{\lambda_{1}} \leq \underline{u}_{\lambda_{2}}$ in $\mathbb{B}$. Therefore, $d \underline{u}_{\lambda} / d \lambda \geq 0$ for all $x \in \mathbb{B}$.

Finally, by differentiating $(1)_{\lambda}$ with respect to $\lambda$ and since $\lambda \rightarrow \underline{u}_{\lambda}$ is nondecreasing, we get

$$
\begin{aligned}
-\Delta^{2} \frac{d \underline{u} \lambda}{d \lambda}-\frac{\lambda}{\left(1-\underline{u}_{\lambda}\right)^{2}} \frac{d \underline{u}_{\lambda}}{d \lambda}=\frac{\lambda}{1-\underline{u}_{\lambda}} \geq 0, \\
x \in \mathbb{B} ; \quad \frac{d \underline{u}_{\lambda}}{d \lambda}=0, \quad x \in \partial \mathbb{B} .
\end{aligned}
$$

Applying the strong maximum principle, we conclude that $d \underline{u}_{\lambda} / d \lambda>0$ on $\mathbb{B}$ for all $0<\lambda<\lambda_{*}$.

That $\lambda \rightarrow \mu_{1, \lambda}$ is decreasing follows easily from the variational characterization of $\mu_{1, \lambda}$, the monotonicity of $\lambda \rightarrow$ $\underline{u}_{\lambda}$, and the monotonicity of $\left(1-\underline{u}_{\lambda}\right)^{-2}$ with respect to $\underline{u}_{\lambda}$, and the proof of (ii) is completed.

Now, we give the proof of (iii). Let $\underline{u}_{\lambda}$ be the minimal solution for $(1)_{\lambda}$ so that $\tilde{u}_{\lambda} \geq \underline{u}_{\lambda}$. If the linearization around $\tilde{u}_{\lambda}$ had nonnegative first eigenvalue, then Lemma 17 would also yield $\widetilde{u}_{\lambda} \leq \underline{u}_{\lambda}$ so that $\widetilde{u}_{\lambda}$ and $\underline{u}_{\lambda}$ necessarily coincide, a contradiction.

\subsection{Weak Solutions versus Classical Solutions}

Lemma 19. Let $u_{\mu}$ be a weak solution of $(1)_{\mu}$ with $\mu<\lambda^{*}$. Then, for $\varepsilon>0$ sufficiently small, the problem $(1)_{(1-\varepsilon) \mu}$ possesses a classical solution.

Proof. Let $\tilde{u} \in L^{1}(\mathbb{B})$ be the unique solution of

$$
\int_{\mathbb{B}} \tilde{u} \Delta^{2} \varphi=\mu \int_{\mathbb{B}} \frac{(1-\varepsilon)}{1-u_{\mu}} \varphi d x, \quad \varphi \in C^{4}(\overline{\mathbb{B}}) \cap H_{0}^{2}(\mathbb{B}),
$$

provided by Lemma 15. By hypothesis, we have

$$
\int_{\mathbb{B}} u_{\mu} \Delta^{2} \varphi d x=\mu \int_{\mathbb{B}} \frac{1}{1-u_{\mu}} \varphi d x, \quad \varphi \in C^{4}(\overline{\mathbb{B}}) \cap H_{0}^{2}(\mathbb{B}) .
$$

By uniqueness, we get

$$
(1-\varepsilon) u_{\mu}=\widetilde{u}
$$

whereas Lemma 13 yields $\tilde{u}>0$ almost everywhere in $\mathbb{B}$ and hence we may assume

$$
u_{\mu}>\tilde{u}, \quad x \in \mathbb{B} \backslash\{x \in \mathbb{B}: \tilde{u}=0\} .
$$

Therefore,

$$
\begin{aligned}
\int_{\mathbb{B}} \tilde{u} \Delta^{2} \varphi & =\int_{\mathbb{B}} \frac{(1-\varepsilon) \mu}{(1-(1 /(1-\varepsilon)) \tilde{u})} d x \\
& \geq(1-\varepsilon) \mu \int_{\mathbb{B}} \frac{1}{1-\tilde{u}} d x, \quad \varphi \in C^{4}(\overline{\mathbb{B}}) \cap H_{0}^{2}(\mathbb{B}) ;
\end{aligned}
$$

thus $\tilde{u}$ is a weak supersolution of $(1)_{(1-\varepsilon) \mu}$ and Proposition 16 yields a weak solution $v$ of $(1)_{(1-\varepsilon) \mu}$ which satisfies

$$
0 \leq v \leq \widetilde{u}<u_{\mu} \leq 1
$$

and then classical by Remark 5 .

Remark 20. From this lemma, we know that $\lambda^{*}=\lambda_{*}$; in what follows, we always denote by $\lambda_{*}$ the largest possible value of $\lambda$ such that $(1)_{\lambda}$ has a solution, unless otherwise stated.

Proposition 21. Up to a subsequence, the convergence

$$
u^{*}:=\lim _{\lambda>\lambda_{*}} \underline{u}_{\lambda}(x)
$$

holds in $H_{0}^{2}(\mathbb{B})$ and the extremal solution $u_{\lambda^{*}}$ satisfies

$$
\int_{\mathbb{B}} \Delta u^{*} \Delta \varphi=\lambda_{*} \int_{\mathbb{B}} \frac{\varphi}{\left(1-u^{*}\right)}, \quad \varphi \in C_{0}^{\infty}(\mathbb{B}) .
$$

In particular, the extremal solution is weakly stable, and if $\left\|u^{*}\right\|_{\infty}<1$, then $\mu_{1}\left(u^{*}\right)=0$.

Proof. Since $\underline{u}_{\lambda}$ is stable, we have

$$
\begin{aligned}
\lambda \int_{\mathbb{B}} \frac{\underline{u}_{\lambda}^{2}}{\left(1-\underline{u}_{\lambda}\right)^{2}} d x & \leq \int_{\mathbb{B}}\left|\Delta \underline{u}_{\lambda}\right|^{2} d x \\
& =\int_{\mathbb{B}} \underline{u}_{\lambda} \Delta^{2} \underline{u}_{\lambda}=\lambda \int_{\mathbb{B}} \frac{\underline{u}_{\lambda}}{1-\underline{u}_{\lambda}} d x .
\end{aligned}
$$

Next, it is easy to check that the following elementary inequality holds: there exists a constant $C>0$ such that

$$
(1+C) \frac{s}{(1-s)} \leq \frac{s^{2}}{(1-s)^{2}}+(1+C), \quad s \in(0,1),
$$

which, used in (60), yields

$$
\lambda \int_{\mathbb{B}} \frac{\underline{u}_{\lambda}}{1-\underline{u}_{\lambda}} \geq \lambda \int_{\mathbb{B}} \frac{\underline{u}_{\lambda}^{2}}{\left(1-\underline{u}_{\lambda}\right)^{2}} d x \geq \lambda(1+C) \int_{\mathbb{B}} \frac{\underline{u}_{\lambda}}{1-\underline{u}_{\lambda}}-C_{1},
$$

where $C_{1}$ is independent of $\lambda$. From the above inequality, we get

$$
\left\|\Delta \underline{u}_{\lambda}\right\|_{2}^{2}=\lambda \int_{\mathbb{B}} \frac{\underline{u}_{\lambda}}{1-\underline{u}_{\lambda}} d x \leq C
$$


Therefore, we may assume $\underline{u}_{\lambda} \rightarrow u^{*}$ in $H_{0}^{2}(\mathbb{B})$ and, by monotone convergence theorem (59), holds after integration by parts. Since $\mu_{1}\left(\underline{u}_{\lambda}\right)>0$ for all $\lambda \in\left(0, \lambda_{*}\right)$, in particular we have

$$
\int_{\mathbb{B}}|\Delta \varphi|^{2} d x \geq \int_{\mathbb{B}} \frac{\lambda \varphi^{2}}{\left(1-\underline{u}_{\lambda}\right)^{2}}, \quad \varphi \in C_{0}^{\infty}(\mathbb{B}),
$$

and passing to the limit as $\lambda \nearrow \lambda_{*}$, we obtain that $u_{\lambda_{*}}$ is weakly stable. Finally, if $\left\|u_{\lambda_{*}}\right\|_{\infty}<1$ and hence $u_{\lambda_{*}}$ is a classical solution of $(1)_{\lambda_{*}}$, the linearized operator at $u_{\lambda_{*}}$

$$
L\left(\lambda_{*}, u_{\lambda_{*}}\right):=\Delta^{2}-\frac{\lambda_{*}}{\left(1-u_{\lambda_{*}}\right)^{2}}
$$

is well defined on the space $\mathbb{R}^{+} \times C^{4, \alpha}(\mathbb{B})$. If $\mu_{1}\left(u_{\lambda_{*}}\right)>0$, then the Implicit Function Theorem applied to the function

$$
F\left(\lambda, u_{\lambda}\right):=\Delta^{2} u_{\lambda}-\frac{\lambda}{1-u_{\lambda}}
$$

would yield a solution for $\lambda>\lambda_{*}$ contradicting the definition of $\lambda_{*}$; thus $\mu_{1}\left(u^{*}\right)=0$.

Corollary 22. There exists a constant $C$ independent of $\lambda$ such that, for each $\lambda \in\left(0, \lambda_{*}\right)$, the minimal solution $\underline{u}_{\lambda}$ satisfies $\left\|\left(1-\underline{u}_{\lambda}\right)^{-1}\right\|_{L^{2}} \leq C$.

Proof. From Proposition 21, we have

$$
\begin{aligned}
\int_{\mathbb{B}} \frac{\underline{u}_{\lambda}^{2}}{\left(1-\underline{u}_{\lambda}\right)^{2}} d x= & \int_{\underline{u}_{\lambda} \geq 1 / 2} \frac{\underline{u}_{\lambda}^{2}}{\left(1-\underline{u}_{\lambda}\right)^{2}} d x \\
& +\int_{\underline{u}_{\lambda}<1 / 2} \frac{\underline{u}_{\lambda}^{2}}{\left(1-\underline{u}_{\lambda}\right)^{2}} d x \leq C .
\end{aligned}
$$

So

$$
\int_{\underline{u}_{\lambda} \geq 1 / 2} \frac{1}{\left(1-\underline{u}_{\lambda}\right)^{2}} d x \leq 4 \int_{\underline{u}_{\lambda} \geq 1 / 2} \frac{\underline{u}_{\lambda}^{2}}{\left(1-\underline{u}_{\lambda}\right)^{2}} d x \leq C .
$$

From this, we easily obtain $\left\|\left(1-\underline{u}_{\lambda}\right)^{-1}\right\|_{L^{2}} \leq C$, and the proof is completed.

Corollary 23. For dimensions $n \leq 4$, the extremal solution $u^{*}$ is regular; that is, $u^{*}=\lim _{\lambda>\lambda_{*}} u_{\lambda}$ exists in the topology of $C^{4}(\mathbb{B})$.

Proof. Since $u^{*}$ is radial and radially decreasing, we need just to show that $u^{*}(0)<1$ to get the regularity of $u^{*}$. Since $\left(1-u^{*}(x)\right) \in L^{2}(\mathbb{B})$ according to Corollary 22, we have that $u^{*}(x) \in W^{4,2}(\mathbb{B})$ by the standard elliptic regularity theory. And then by the Sobolev imbedding theorem, we have $u^{*}(x) \in C^{4-[n / 8]-1,[n / 8]+1-(n / 8)}(\mathbb{B})$. So if $n \leq 4$, one can easily see that $u^{*}(x) \in C^{2}(\mathbb{B})$. As $\nabla u^{*}(0)=0$, we get

$$
1-u^{*}(x)=u^{*}(0)-u^{*}(x) \leq C|x|^{2} ;
$$

hence

$$
\infty>\int_{\mathbb{B}} \frac{d x}{\left(1-u^{*}(x)\right)^{2}} \geq C \int_{\mathbb{B}} \frac{d x}{|x|^{4}}=\infty .
$$

A contradiction arises, so $u^{*}$ is regular for $n \leq 4$.

\subsection{The Upper and Lower Bounds for $\lambda_{*}$}

Lemma 24. Consider

$$
\lambda_{*} \leq \frac{\nu_{1}}{4}
$$

where $v_{1}$ is the first eigenvalue of $\Delta^{2}$ in $H_{0}^{2}(\mathbb{B})$.

Proof. Let $\underline{u}_{\lambda}$ be a solution of $(1)_{\lambda}$ and let $\left(\psi, v_{1}\right)$ denote the first eigenpair of $\Delta^{2}$ in $H_{0}^{2}(\mathbb{B})$ with $\psi>0$; then

$$
\nu_{1} \int_{\mathbb{B}} \underline{u}_{\lambda} \psi d x=\int_{\mathbb{B}} \underline{u}_{\lambda} \Delta^{2} \psi d x=\lambda \int_{\mathbb{B}} \frac{\psi}{1-\underline{u}_{\lambda}}
$$

and this implies

$$
\int_{\mathbb{B}}\left(-v_{1} \underline{u}_{\lambda}+\frac{\lambda}{1-\underline{u}_{\lambda}}\right) \psi d x=0 .
$$

Since $\psi>0$, there must exist a point $\bar{x} \in \mathbb{B}$ where

$$
\frac{\lambda}{1-\underline{u}_{\lambda}}-v_{1} \underline{u}_{\lambda} \leq 0 .
$$

And one can conclude that $\lambda_{*} \leq \sup _{0 \leq \underline{u}_{\lambda} \leq 1} \nu_{1} \underline{u}_{\lambda}\left(1-\underline{u}_{\lambda}\right)=$ $v_{1} / 4$.

The lower bound for $\lambda_{*}$ is obtained by finding a suitable supersolution. For example, if for some parameter $\tilde{\lambda}_{1}$ there exists a supersolution, then $\lambda_{*}>\widetilde{\lambda}_{1}$ by Proposition 18 .

Lemma 25. For $n \geq 1$, one has

$$
\lambda_{*} \geq \max \{4 n(n-2), 2 n(n+2)\} \text {. }
$$

Proof. For any $\beta>0$ and $C_{0}>0$, let $g_{\beta}(r)=\left(C_{0}-\log r\right)^{\beta}, r \in$ $(0,1)$. Then, by direct calculation, we find the following facts:

$$
\begin{gathered}
\Delta g_{\beta}(r)=\beta r^{-2}\left[(2-n) g_{\beta-1}+(\beta-1) g_{\beta-2}\right], \\
\Delta\left[r^{2} g_{\beta}\right]=2 n g_{\beta}-\beta(n+2) g_{\beta-1}+\beta(\beta-1) g_{\beta-2} .
\end{gathered}
$$

So we have

$$
\begin{aligned}
\Delta^{2}\left(r^{2} g_{\beta}\right)= & 2 n \Delta g_{\beta}-\beta(n+2) \Delta g_{\beta-1}(r) \\
& +\beta(\beta-1) \Delta g_{\beta-2}(r) \\
= & \beta r^{-2} \times\left\{2 n(2-n) g_{\beta-1}(r)\right. \\
& \left.+(\beta-1)\left(2 n+n^{2}-4\right) g_{\beta-2}(r)\right\} \\
+ & \beta r^{-2} \times\left\{(\beta-1)(\beta-2) \times(-2 n) g_{\beta-3}(r)\right. \\
& \left.+(\beta-1)(\beta-2)(\beta-3) g_{\beta-4}\right\} .
\end{aligned}
$$


Now, let $\beta \in(0,1)$ and $n>2$; we have

$$
\Delta^{2}\left(r^{2} g_{\beta}\right) \leq \beta r^{-2} \times 2 n(n-2) g_{\beta-1} .
$$

Also for any $A>0$ take $\bar{u}=1-A r^{2} g_{\beta}$; one concludes from (78) that

$$
\Delta^{2} u \geq 2 n(n-2) A \beta r^{-2} g_{\beta-1}
$$

Set $\beta=1 / 2$; one can obtain that

$$
\begin{aligned}
\Delta^{2} \bar{u} & \geq \frac{n(n-2) A^{2}}{1-\bar{u}} \text { in } \mathbb{B}_{1}, \\
\bar{u}(r) & =1-C_{0}^{1 / 2} A \quad \text { on } \partial \mathbb{B}_{1}, \\
\bar{u}^{\prime}(r) & =A C_{0}^{-1 / 2}\left(\frac{1}{2}-2 C_{0}\right) \quad \text { on } \partial \mathbb{B}_{1} .
\end{aligned}
$$

Choosing $C_{0}=1 / 4, A_{0}=2$, one concludes that $\bar{u}(r)$ is a supersolution of $(1)_{4 n(n-2)}$ and $\lambda_{*} \geq 4 n(n-2)$ according to Proposition 18. Besides, we consider the function

$$
\omega_{\alpha}(x):=\alpha\left(1-|x|^{2}\right)^{2}, \quad \alpha \in(0,1),
$$

which satisfies $0 \leq \omega_{\alpha}(x)<1$ for $x \in \mathbb{B}$ and

$$
\omega_{\alpha}(x)=0, \quad \frac{\partial \omega_{\alpha}}{\partial n}=0 \quad \text { for } x \in \partial \mathbb{B} ; \forall \alpha \in(0,1)
$$

Now, the idea is to obtain from $\omega_{\alpha}(x)$ a supersolution of $(1)_{\lambda}$, for a suitable choice of $\alpha$ and for $\lambda$ in a suitable range of the form $0<\lambda \leq \tilde{\lambda}$. For simple calculation, we have

$$
\begin{aligned}
\Delta^{2} \omega_{\alpha}(r)= & \frac{d^{4} \omega_{\alpha}}{d r^{4}}+\frac{2(n-1)}{r}+\frac{d^{3} \omega_{\alpha}}{d r^{3}} \\
& +\frac{(n-)(n-3)}{r^{2}} \frac{d^{2} \omega_{\alpha}}{d r^{2}}-\frac{(n-1)(n-3)}{r^{3}} \frac{d \omega_{\alpha}}{d r} \\
= & {\left[8 n^{2}+16 n\right] \alpha=: C(n) \alpha, }
\end{aligned}
$$

and thus

$$
\begin{aligned}
\Delta^{2} \omega_{\alpha}(r) & =\frac{C(n) \alpha(1-\alpha)}{1-\alpha} \geq \frac{C(n) \alpha(1-\alpha)}{\left[1-\alpha\left(1-|x|^{4}\right)\right]} \\
& =\frac{C(n) \alpha(1-\alpha)}{1-\omega_{\alpha}}
\end{aligned}
$$

from which we deduce that

$$
\lambda_{*}=\lambda^{*} \geq \sup _{\alpha \in(0,1)} C(n) \alpha(1-\alpha)=\frac{1}{4} C(n)=2 n(n+2),
$$

and the proof is completed. and 8.

We complete this section by giving proofs of Theorems 7

Proofs of Theorems 7 and 8. The proof of Theorem 7 follows from Proposition 18 and Lemmas 24 and 25. For the proof of Theorem 8 , we only need to prove the uniqueness of the regular extremal solution $u^{*}$; the other parts of Theorem 8 follow from Lemma 19 and Corollary 23. Indeed, if the extremal solution $u^{*}$ is regular, we can easily check that $\mu_{1}\left(u^{*}\right)=0$ by Implicit Function Theorem, since, otherwise, we can continue the minimal branch beyond $\lambda_{*}$. And then the uniqueness follows from (ii) of the Lemma 17.

\section{Uniqueness of the Extremal Solution: Proof of Theorem 9}

Proof of Theorem 9. Suppose that $v \in H^{2}(\mathbb{B})$ satisfies

$$
\begin{gathered}
\int_{\mathbb{B}} v \Delta^{2} \varphi d x \geq \int_{\mathbb{B}} \frac{\lambda_{*}}{1-v} d x, \quad \forall \varphi \in C_{0}^{\infty}(\overline{\mathbb{B}}), \varphi \geq 0, \\
v\left|\partial \mathbb{B}=0, \quad \frac{\partial v}{\partial n}\right|_{\partial \mathbb{B}} \leq 0,
\end{gathered}
$$

and $v \quad \equiv \quad u^{*}$. Notice that the construction of minimal solutions in Proposition 18 for $\lambda \in\left(0, \lambda_{*}\right)$ carries over to $\lambda=\lambda_{*}$, but just in the weak sense; precisely, we may assume that for $\lambda=\lambda^{*}$ there exists a minimal weak solution. In other words, it is legitimate to assume

$$
v(x) \geq u^{*}, \quad \text { almost everywhere } x \in \mathbb{B} \text {. }
$$

The idea of the proof is as follows: first, we prove that the function

$$
u_{0}=\frac{1}{2}\left(u^{*}+v\right)
$$

is a supersolution to the following perturbation of problem $(1)_{\lambda}$ :

$$
\begin{gathered}
\Delta^{2} u=\frac{\lambda_{*}}{1-u}+\mu \frac{\zeta(x)}{1-u}, \quad \text { in } \mathbb{B} ; \\
0 \leq u \leq 1, \quad \text { in } \mathbb{B} ; \\
u=\frac{\partial u}{\partial n}=0, \quad \text { on } \partial \mathbb{B}
\end{gathered}
$$

for a standard cut-off function $\zeta(x) \in C_{0}^{\infty}(\mathbb{B})$ and $\mu>0$ to be suitably chosen; besides, a solution is understood in weak sense unless otherwise stated. Second, we construct, for some $\lambda>\lambda_{*}$, a supersolution to (1) $\lambda$ by using a solution of (89) and this will enable us to build up a weak solution of $(1)_{\lambda}$ for $\lambda>\lambda_{*}$ and thus necessarily $v \equiv u^{*}$.

Indeed, we first observe that for $0<R<1$ and for some $c_{0}=c_{0}(R)>0$

$$
v(x) \geq u^{*}+c_{0} \quad|x| \leq R .
$$

To prove this, we recall Green's function for $\Delta^{2}$ with Dirichlet boundary conditions

$$
\begin{gathered}
\Delta_{x}^{2} G(x, y)=\delta_{y}, \quad x \in \mathbb{B} ; \\
G(x, y)=0, \quad x \in \partial \mathbb{B} ; \\
\frac{\partial G}{\partial n}(x, y)=0, \quad x \in \partial \mathbb{B},
\end{gathered}
$$


where $\delta_{y}$ is the Dirac mass at $y \in \mathbb{B}$. Boggio gave an explicit formula for $G(x, y)$ which was used in [17] to prove that in dimension $n \geq 5$

$$
G(x, y) \sim|x-y|^{4-n} \min \left(1, \frac{d(x)^{2} d(y)^{2}}{|x-y|^{4}}\right),
$$

where

$$
d(x)=\operatorname{dist}(x, \partial \mathbb{B})=1-|x| .
$$

Formula (92) yields

$$
G(x, y) \geq c d(x)^{2} d(y)^{2}
$$

for some $c>0$, and this in turn implies that, for smooth functions $\bar{v}$ and $\bar{u}$ such that $\bar{v}-\bar{u} \in H_{0}^{2}(\mathbb{B})$ and $\Delta^{2}(\bar{v}-\bar{u}) \geq 0$,

$$
\begin{aligned}
\widetilde{v}-\tilde{u}= & \int_{\partial B}\left(\frac{\partial \Delta_{x} G}{\partial n_{x}}(x, y) \widetilde{v}-\widetilde{u}-\Delta_{x} G(x, y) \frac{\partial(\widetilde{v}-\widetilde{u})}{\partial n}\right) \\
& +\int_{B} G(x, y) \Delta^{2}(\widetilde{v}-\widetilde{u}) d x \\
\geq & c d(y)^{2} \int_{B} \Delta^{2}(\widetilde{v}-\tilde{u}) d(x)^{2} d x .
\end{aligned}
$$

Using a standard approximation procedure, we conclude that

$$
v(y)-u^{*}(y) \geq c d(y)^{2} \lambda^{*} \int_{\mathbb{B}}\left(\frac{1}{1-v}-\frac{1}{1-u^{*}}\right) d(x)^{2} d x .
$$

Since $v \geq u^{*}, v \not \equiv u^{*}$, we deduce (92).

Let $u_{0}=\left(u^{*}+v\right) / 2$. Then, by Taylor's theorem,

$$
\begin{aligned}
\frac{1}{1-v}= & \frac{1}{1-u_{0}}+\frac{v-u_{0}}{\left(1-u_{0}\right)^{2}}+\frac{\left(v-u_{0}\right)^{2}}{4\left(1-u_{0}\right)^{3}} \\
& +\frac{\left(v-u_{0}\right)^{3}}{18\left(1-u_{0}\right)^{3}}+\frac{\left(v-u_{0}\right)^{4}}{96\left(1-\varepsilon_{1}\right)^{4}}
\end{aligned}
$$

for some $u_{0} \leq \varepsilon_{1} \leq v$ and

$$
\begin{aligned}
\frac{1}{1-u^{*}}= & \frac{1}{1-u_{0}}+\frac{u^{*}-u_{0}}{\left(1-u_{0}\right)^{2}}+\frac{\left(u^{*}-u_{0}\right)^{2}}{4\left(1-u_{0}\right)^{3}} \\
& +\frac{\left(u^{*}-u_{0}\right)^{3}}{18\left(1-u_{0}\right)^{3}}+\frac{\left(u^{*}-u_{0}\right)^{4}}{96\left(1-\varepsilon_{2}\right)^{4}}
\end{aligned}
$$

for some $u^{*} \leq \varepsilon_{2} \leq u_{0}$. Adding (97) and (98) yields

$$
\frac{1}{2}\left(\frac{1}{1-v}+\frac{1}{1-u^{*}}\right) \geq \frac{1}{1-u_{0}}+\frac{1}{16} \frac{\left(u^{*}-v\right)^{2}}{\left(1-u_{0}\right)^{2}}
$$

and in turn we obtain

$$
\begin{aligned}
\int_{\mathbb{B}} u_{0} \Delta^{2} \varphi d x & \geq \int_{\mathbb{B}}\left[\frac{\lambda_{*}}{1-u_{0}}+\frac{\lambda_{*}\left(u^{*}-v\right)^{2}}{16\left(1-u_{0}\right)}\right] d x \\
& \geq \int_{\mathbb{B}}\left[\frac{\lambda_{*}}{1-u_{0}}+\frac{\lambda_{*} c_{0}^{2} \zeta(x)}{16\left(1-u_{0}\right)}\right] d x .
\end{aligned}
$$

Thus, $u_{0}$ is a weak supersolution of (89) with $\mu=\lambda_{*} c_{0}^{2} / 16$ and the cut-off $\zeta(x)$ with support in $\mathbb{B}_{\rho}$. Now, reasoning as in Lemma 19, we may assume, for $\varepsilon>0$ sufficiently small, that (89) possesses a classical solution $0 \leq u_{\varepsilon}<1$ with parameter $\lambda_{*}$ replaced by $\lambda_{*}-\varepsilon$. Set $\mu_{\varepsilon}:=\left[\left(\lambda_{*}-\varepsilon\right) c_{0}^{2}\right] / 16$ and let $\psi \epsilon$ $C^{4}(\overline{\mathbb{B}})$ be the unique classical solution of the following:

$$
\begin{gathered}
\Delta^{2} \psi=\mu_{\varepsilon} \frac{\zeta(x)}{1-u_{\varepsilon}} \text { in } \mathbb{B}, \\
\psi=\frac{\partial \psi}{\partial n}=\text { on } \partial \mathbb{B} .
\end{gathered}
$$

We also, by the Boggio principle, have that there exists $M>0$ sufficiently large such that $u_{\varepsilon} \leq M \psi$. Next, let $\delta>0$ and set

$$
\omega:=\frac{\left(\lambda_{*}-\varepsilon\right)+\delta}{\lambda_{*}-\varepsilon} u_{\varepsilon}-\psi .
$$

Choosing $\delta$ sufficiently small, we obtain $\omega \leq u_{\varepsilon}<1$; moreover, from

$$
\begin{gathered}
\Delta^{2}\left(u_{\varepsilon}-\psi\right)=\left(\lambda_{*}-\varepsilon\right) \frac{1}{1-u_{\varepsilon}} \geq 0, \quad \text { in } \mathbb{B}, \\
u_{\varepsilon}-\psi=\frac{\partial\left(u_{\varepsilon}-\psi\right)}{\partial n}=0 \quad \text { on } \partial \mathbb{B},
\end{gathered}
$$

we have again by the Boggio principle that $\psi \leq u_{\varepsilon}$ and eventually that $\omega \geq 0$. Finally, we have

$$
\begin{aligned}
\Delta^{2} \omega= & \left(\lambda_{*}-\varepsilon+\delta\right) \frac{1}{1-u_{\varepsilon}}+\frac{\left(\lambda_{*}-\varepsilon+\delta\right) c_{0}^{2}}{16} \frac{\varepsilon(x)}{1-u_{\varepsilon}} \\
& -\mu_{\varepsilon} \frac{\varepsilon(x)}{1-u_{\varepsilon}} \geq\left(\lambda_{*}-\varepsilon+\delta\right) \frac{1}{1-\omega}
\end{aligned}
$$

since $\omega \leq u_{\varepsilon}$. Thus, it is enough to choose $0<\varepsilon<\delta$ to provide a classical solution to $(1)_{\lambda}$ for $\lambda>\lambda_{*}$, which is a contradiction; this completes the proof of Theorem 9 .

\section{Behavior of the Minimal Solutions as $\lambda \rightarrow 0$ : Proof of Theorem 11}

Proof of Theorem 11. We first show that

$$
\underline{u}_{\lambda} \longrightarrow 0 \quad \text { uniformly as } \lambda \longrightarrow 0 \text {. }
$$

Since this standard, we just briefly sketch its proof. By Theorem 7, we know that

$$
0<\lambda<\mu<\lambda_{*} \Longrightarrow \underline{u}_{\lambda}(x)<\underline{u}_{\mu}(x) \quad \text { if }|x|<1 .
$$

Then, by multiplying $(1)_{\lambda}$ by $\underline{u}_{\lambda}$ and by integrating by parts, we obtain that $\left\|\underline{u}_{\lambda}\right\|_{H_{0}^{2}(\mathbb{B})}$ remains bounded. Hence, up to a subsequence, $\left\{\underline{u}_{\lambda}\right\}$ converges in the weak $H_{0}^{2}(\mathbb{B})$ topology to 0 , which is the unique solution of $(1)_{0}$. By convergence of the norms, we infer that the convergence is in the norm topology.

Next, note that $U_{\lambda}$ satisfies

$$
\begin{gathered}
\Delta^{2} U_{\lambda}=\lambda \text { in } \mathbb{B}, \\
U_{\lambda}=\frac{\partial U_{\lambda}}{\partial n}=\text { on } \partial \mathbb{B} .
\end{gathered}
$$


Therefore, $\Delta^{2} \underline{u}_{\lambda}>\Delta^{2} U_{\lambda}$; one concludes that $\underline{u}_{\lambda}>U_{\lambda}$ by Lemma 13.

In order to prove the last statement of Theorem 11, note that from (106) we know that for all $\varepsilon>0$ there exists $\lambda_{\varepsilon}>0$ such that $\lambda<\lambda_{\varepsilon} \Rightarrow\left\|\underline{u}_{\lambda}\right\|_{\infty}<\varepsilon$.

So, fix $\varepsilon>0$ and let $\lambda<\lambda_{\varepsilon}$. Then,

$$
\Delta^{2} \underline{u}_{\lambda}=\frac{\lambda}{1-\underline{u}_{\lambda}}<\frac{\lambda}{1-\varepsilon}=\Delta^{2} \frac{U_{\lambda}}{1-\varepsilon} .
$$

This shows that $\underline{u}_{\lambda}(x)<U_{\lambda}(x) /(1-\varepsilon)$ for all $x \in \mathbb{B}$, and the proof is completed according to the arbitrariness of $\varepsilon$.

\section{Further Results and Open Problems}

First, we give the following result which is the main tool to guarantee that $u^{*}$ is singular. At the same time, it gives a precise estimate for $\lambda_{*}$. The proof of this result is based on an upper estimate of $u^{*}$ by a stable singular subsolution.

Proposition 26. Suppose there exist $\lambda^{\prime}>0, \beta>0$ and $a$ singular radial function $\omega(r) \in H_{0}^{2}(\mathbb{B})$ with $1 /(1-\omega(r)) \in$ $L_{\text {loc }}^{\infty}(\overline{\mathbb{B}} \backslash 0)$ such that

$$
\begin{aligned}
\Delta^{2} \omega & \leq \frac{\lambda^{\prime}}{1-\omega} \text { for } 0<r<1, \\
\omega(1) & =\omega^{\prime}(1)=0, \\
\beta \int_{\mathbb{B}} \frac{\phi^{2}}{(1-\omega)^{2}} & \leq \int_{\mathbb{B}}(\Delta \phi)^{2} \quad \forall \phi \in H_{0}^{2}(\mathbb{B}) .
\end{aligned}
$$

If $\beta>\lambda^{\prime}$, then $\lambda_{*}<\lambda^{\prime}$ and $u^{*}$ is singular.

Proof. First, note that (111) and $1 /(1-\omega(r)) \in L_{\mathrm{loc}}^{\infty}(\overline{\mathbb{B}} \backslash 0)$ yield $1 /(1-\omega) \in L^{1}(\mathbb{B})$. Equation (110) implies that $\omega(r)$ is a $H_{0}^{2}(\mathbb{B})$-weak subsolution of $(1)_{\lambda^{\prime}}$. If now $\lambda^{\prime}<\lambda^{*}$, then by Lemma $17 \omega(r)$ would necessarily be below the minimal solution $\underline{u}_{\lambda^{\prime}}$, which is a contradiction since $\omega(r)$ is singular while $\underline{u}_{\lambda^{\prime}}$ is regular. In the following, we will prove that $u^{*}$ is singular.

Now, let $\lambda^{\prime} / \beta<\gamma<1$ in such a way that

$$
\alpha:=\left(\frac{\gamma \lambda_{*}}{\lambda^{\prime}}\right)^{1 / 2}<1 .
$$

Setting $\bar{\omega}:=1-\alpha(1-\omega)$, we claim that

$$
u^{*} \leq \bar{\omega} \quad \text { in } \mathbb{B}
$$

Note that by the choice of $\alpha$ we have $\alpha^{2} \lambda^{\prime}<\lambda_{*}$, and therefore to prove (113) it suffices to show that, for $\alpha^{2} \lambda^{\prime} \leq \lambda<\lambda_{*}$, we have $u_{\lambda} \leq \bar{\omega}$ in $\mathbb{B}$. Indeed, fix such $\lambda$ and note that

$$
\Delta^{2} \bar{\omega}=\alpha \Delta^{2} \omega \leq \frac{\alpha \lambda^{\prime}}{(1-\omega)}=\frac{\alpha^{2} \lambda^{\prime}}{(1-\bar{\omega})} \leq \frac{\lambda}{(1-\bar{\omega})} .
$$

Assume that $\underline{u}_{\lambda} \leq \bar{\omega}$ does not hold in $\mathbb{B}$, and consider

$$
R_{1}:=\sup \left\{0 \leq R \leq 1 \mid \underline{u}_{\lambda}(R)>\bar{\omega}(R)\right\}>0 .
$$

Since $\bar{\omega}(1)=1-\alpha>0=u_{\lambda}(1)$, we then have

$$
R_{1}<1, \quad u_{\lambda}\left(R_{1}\right)=\bar{\omega}\left(R_{1}\right), \quad \underline{u}_{\lambda}^{\prime}\left(R_{1}\right) \leq \bar{\omega}^{\prime}\left(R_{1}\right) .
$$

Now, consider the following problem:

$$
\begin{aligned}
\Delta^{2} u & =\frac{\lambda}{1-u} \quad \text { in } \mathbb{B}_{R_{1}}, \\
u & =u_{\lambda}\left(R_{1}\right) \quad \text { on } \partial \mathbb{B}_{R_{1}}, \\
\frac{\partial u}{\partial n} & =u_{\lambda}^{\prime}\left(R_{1}\right) \quad \text { on } \partial \mathbb{B}_{R_{1}} .
\end{aligned}
$$

Then, $\underline{u}_{\lambda}$ is a solution to the above problem while $\bar{\omega}$ is a subsolution to the same problem. Moreover, $\bar{\omega}$ is stable since $\lambda<\lambda_{*}$ and

$$
\frac{\lambda}{(1-\bar{\omega})^{2}} \leq \frac{\lambda_{*}}{\alpha^{2}(1-\omega)^{2}}<\frac{\beta}{(1-\omega)^{2}} .
$$

By Lemma 19, we deduce that $\underline{u}_{\lambda} \geq \bar{\omega}$ in $\mathbb{B}_{R_{1}}$ which is impossible, since $\bar{\omega}$ is singular while $u_{\lambda}$ is regular. This establishes claim (113) which, combined with the above inequality, yields

$$
\frac{\lambda_{*}}{\left(1-u_{*}\right)^{2}} \leq \frac{\lambda_{*}}{\alpha^{2}(1-\omega)^{2}}<\frac{\beta}{(1-\omega)},
$$

and thus

$$
\inf _{\varphi \in C_{0}^{\infty}(\mathbb{B})} \frac{\int_{\mathbb{B}}\left[(\Delta \varphi)^{2}-\left(\lambda_{*} \varphi^{2} /\left(1-u^{*}\right)^{2}\right)\right] d x}{\int_{\mathbb{B}} \varphi^{2} d x}>0 .
$$

This is not possible if $u^{*}$ is a smooth function since otherwise one could use the Implicit Function Theorem to continue the minimal branch beyond $\lambda_{*}$. The proof is over.

(i) Open Problem 1. Does (1) $\lambda$ possess a stable singular subsolution? We know that Cowan et al., with the help of Maple, construct such solution of $\left(P_{\lambda}\right)$ with $p=2$ by improved Hardy-Rellich Inequalities; see $[4,9]$. But the method used there seems invalid when $p=1$.

We now turn to the extremal solution $u^{*}$. We suggest the following open problems.

(ii) Open Problem 2. Does one find the precise estimate for $u^{*}$ as in $[4,9,15]$, which plays a crucial role for investigating the regularity of $u^{*}$ ? In [4], the precise bound for $u^{*}$ is obtained by finding a stable singular subsolution which relies on the "ghost" singular solution, as mentioned in the Introduction section. However, in the present paper, we cannot find any "ghost" singular solution, so a new trick is needed.

(iii) Open Problem 3. For the corresponding second equation, the extremal solution $u^{*}$ is regular for dimensions $n \leq 6$ and singular for dimension $n \geq 7$; for details, see [11]. The threshold $n^{*}=7$ between regular and singular solutions is called the critical dimension. There is a natural question: whether there exists a critical dimension $N^{*}$ for $(1)_{\lambda}$. We conjecture that $N^{*}=8$. 


\section{Conflict of Interests}

The author declares that there is no conflict of interests concerning the publication of this paper.

\section{Acknowledgments}

The author would like to thank Professor Dong Ye for his constructive comments. This research was supported by NSFC (nos. 11201119 and 11126155).

\section{References}

[1] J. A. Pelesko and A. A. Bernstein, Modeling MEMS and NEMS, Chapman Hall and CRC Press, 2002.

[2] M. Ghergu, "A biharmonic equation with singular nonlinearity," Proceedings of the Edinburgh Mathematical Society. Series II, vol. 55, no. 1, pp. 155-166, 2012.

[3] D. Cassani, J. M. do Ó, and N. Ghoussoub, "On a fourth order elliptic problem with a singular nonlinearity," Advanced Nonlinear Studies, vol. 9, no. 1, pp. 177-197, 2009.

[4] C. Cowan, P. Esposito, N. Ghoussoub, and A. Moradifam, "The critical dimension for a fourth order elliptic problem with singular nonlinearity," Archive for Rational Mechanics and Analysis, vol. 198, no. 3, pp. 763-787, 2010.

[5] J. Dávila, I. Flores, and I. Guerra, "Multiplicity of solutions for a fourth order equation with power-type nonlinearity," Mathematische Annalen, vol. 348, no. 1, pp. 143-193, 2010.

[6] P. Esposito, N. Ghoussoub, and Y. Guo, Mathematical Analysis of Partial Differential Equations Modeling Electrostatic MEMS, vol. 20 of American Mathematical Society, Courant Institute of Mathematical Sciences, New York, NY, USA, 2010.

[7] Z. Guo and J. Wei, "On a fourth order nonlinear elliptic equation with negative exponent," SIAM Journal on Mathematical Analysis, vol. 40, no. 5, pp. 2034-2054, 2009.

[8] F. Lin and Y. Yang, "Nonlinear non-local elliptic equation modelling electrostatic actuation," Proceedings of The Royal Society of London. Series A. Mathematical, Physical and Engineering Sciences, vol. 463, no. 2081, pp. 1323-1337, 2007.

[9] A. Moradifam, "On the critical dimension of a fourth order elliptic problem with negative exponent," Journal of Differential Equations, vol. 248, no. 3, pp. 594-616, 2010.

[10] T. Boggio, "Sulle funzioni di green d'ordine m," Rendiconti del Circolo Matematico di Palermo, vol. 20, pp. 97-135, 1905.

[11] A. M. Meadows, "Stable and singular solutions of the equation $\Delta u=1 / u$," Indiana University Mathematics Journal, vol. 53, no. 6, pp. 1681-1703, 2004.

[12] S. Agmon, A. Douglis, and L. Nirenberg, "Estimates near the boundary for solutions of elliptic partial differential equations satisfying general boundary conditions. I," Communications on Pure and Applied Mathematics, vol. 12, pp. 623-727, 1959.

[13] H. Brezis, T. Cazenave, Y. Martel, and A. Ramiandrisoa, "Blow up for $u_{t}-\Delta u=g(u)$ revisited," Advances in Differential Equations, vol. 1, no. 1, pp. 73-90, 1996.

[14] Y. Martel, "Uniqueness of weak extremal solutions of nonlinear elliptic problems," Houston Journal of Mathematics, vol. 23, no. 1, pp. 161-168, 1997.

[15] J. Dávila, L. Dupaigne, I. Guerra, and M. Montenegro, "Stable solutions for the bilaplacian with exponential nonlinearity," SIAM Journal on Mathematical Analysis, vol. 39, no. 2, pp. 565592, 2007.
[16] G. Arioli, F. Gazzola, H.-C. Grunau, and E. Mitidieri, "A semilinear fourth order elliptic problem with exponential nonlinearity," SIAM Journal on Mathematical Analysis, vol. 36, no. 4, pp. 1226-1258, 2005.

[17] H.-C. Grunau and G. Sweers, "Positivity for equations involving polyharmonic operators with Dirichlet boundary conditions," Mathematische Annalen, vol. 307, no. 4, pp. 589-626, 1997. 


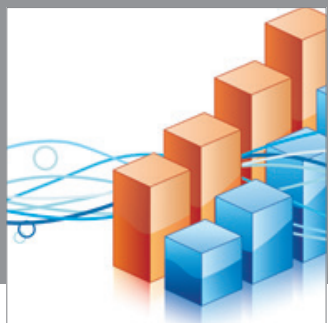

Advances in

Operations Research

mansans

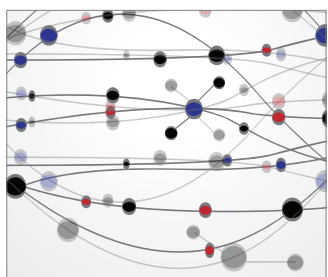

The Scientific World Journal
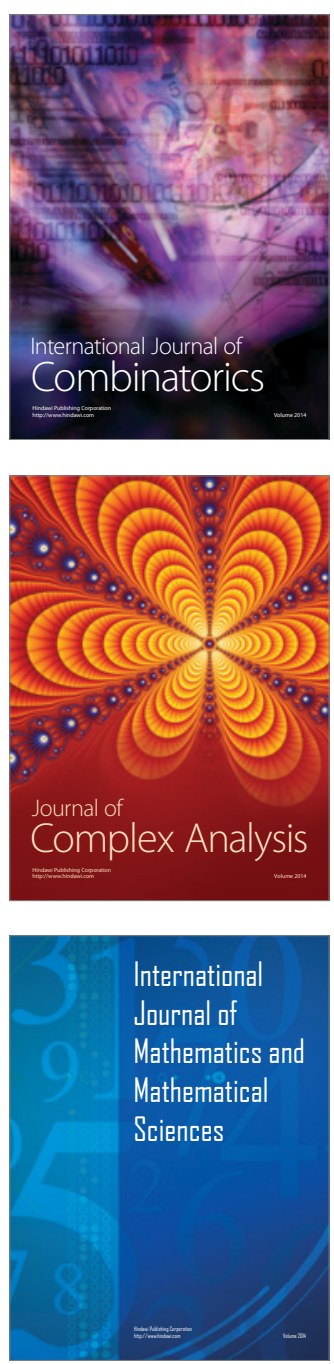
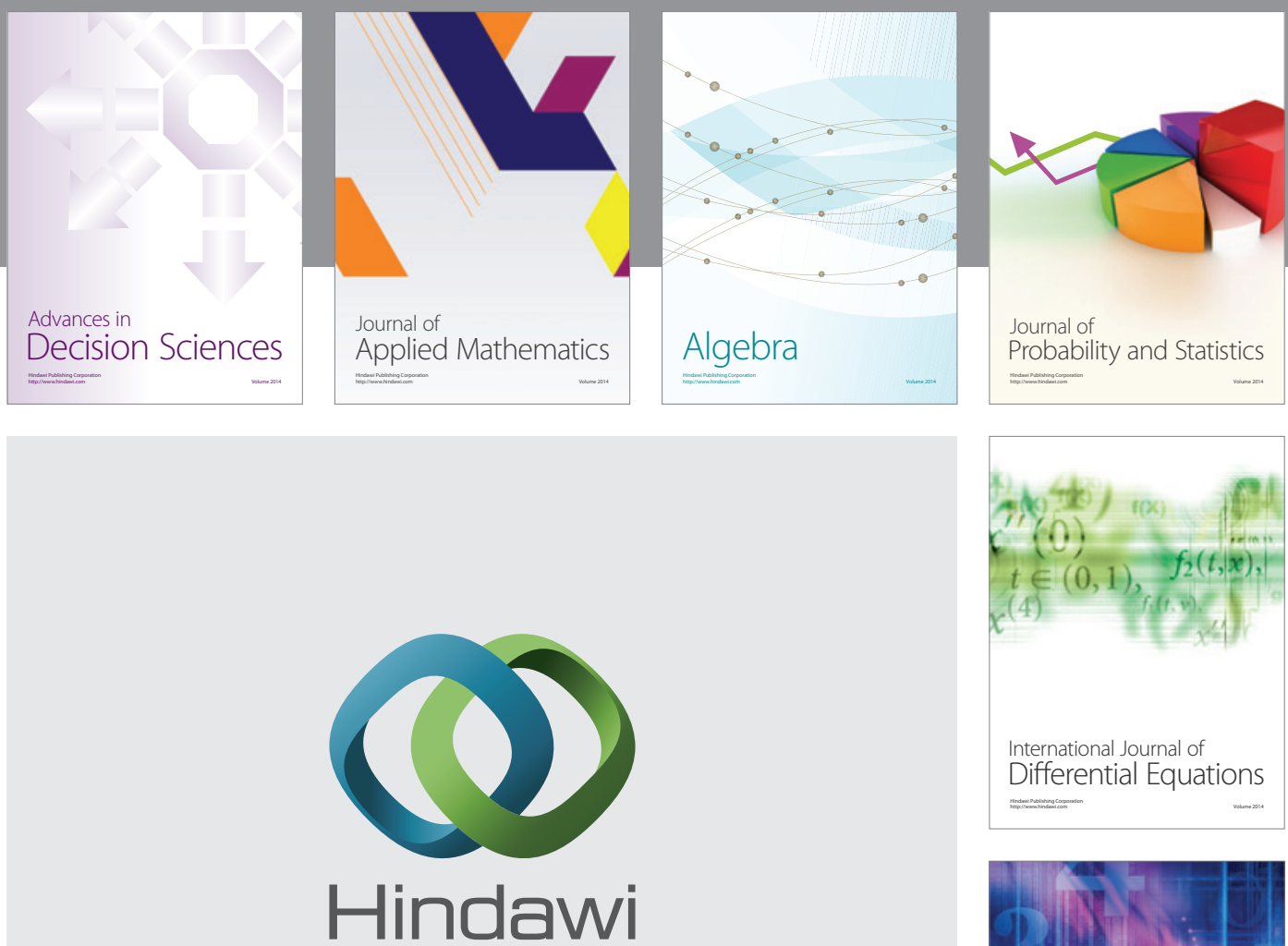

Submit your manuscripts at http://www.hindawi.com
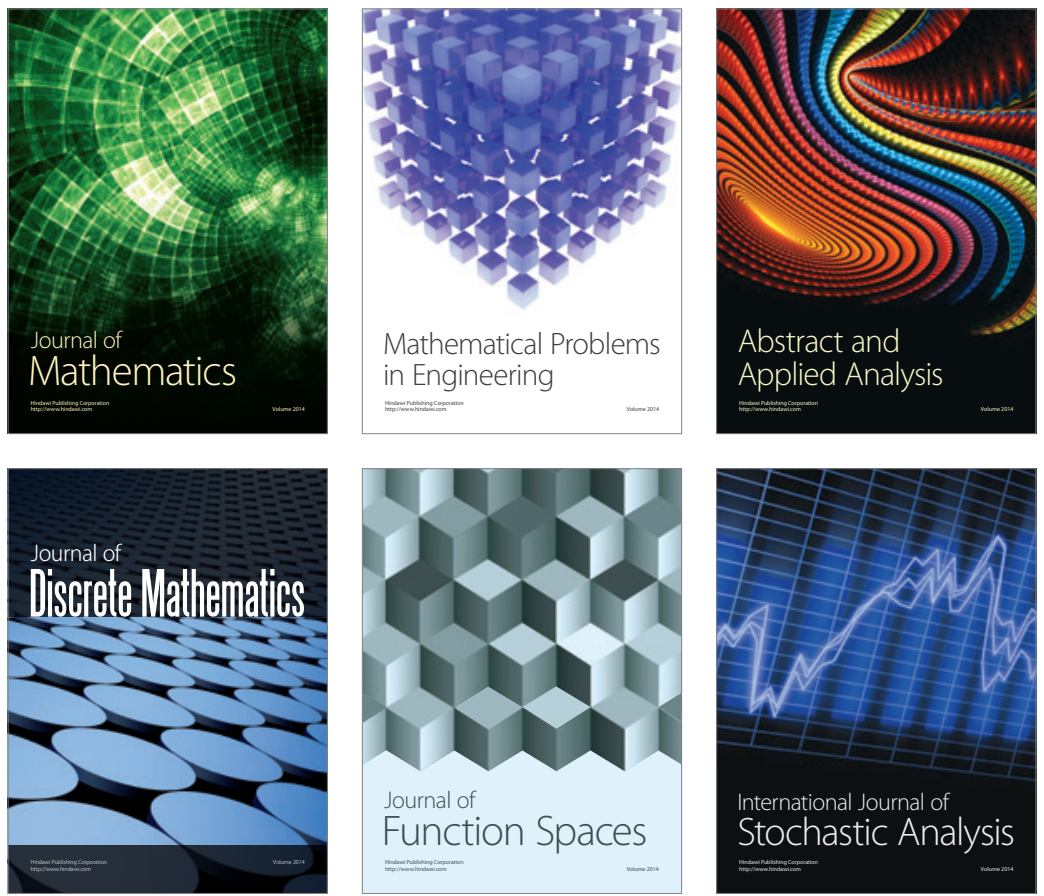

Journal of

Function Spaces

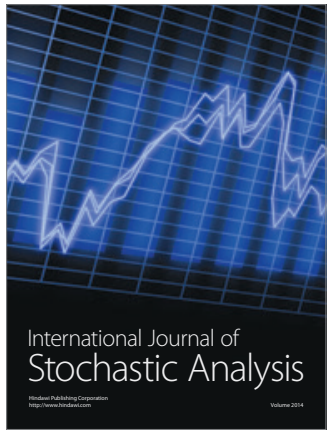

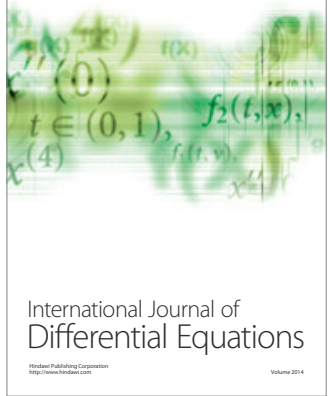
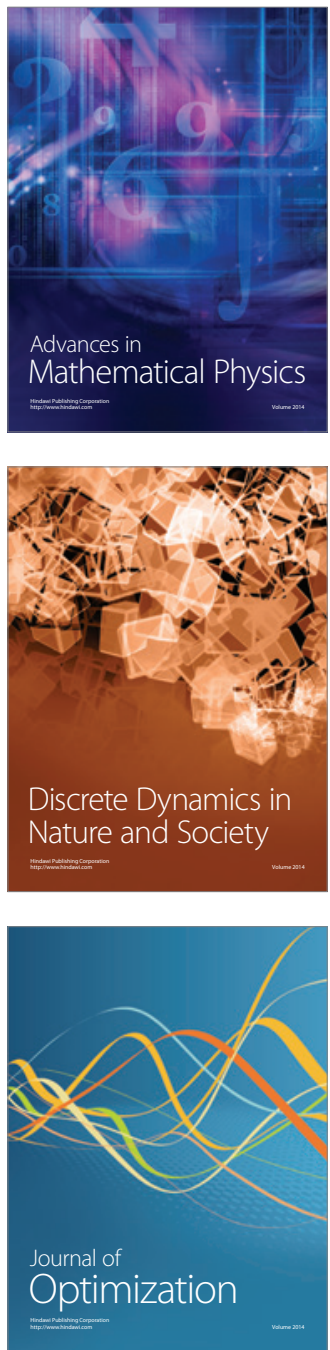